\title{
Self-Insertion of Metal Rodis into Urethra
}

Nerli RB, Abhifith SM, Ajay Kumar G, Dixit NS, Gupta R, Hiremath MB

Department of Urology, KLE University's J N Medical College, KLES Dr Prabhakar

Kore Hospital \& MRC, Belgaum, Karnataka-590010, India.

\section{Abstract:}

Numerous accounts of foreign objects, which sometimes defy imagination and include all types of objects, inserted into the urethra have been documented. The frequency of such cases renders these an important addition to the diseases of the genitourinary organs. Regardless of the motive for insertion, the extraction, can be challenging and requires creative deployment of the full armamentarium of the specialist. We report a 26 year old male with history of insertion of foreign objects into his urethra for sexual stimulation. Plain $X$-ray imaging revealed metallic rods within the urethra. The foreign bodies were extracted per-urethra under general anaesthesia with the use of cystoscopes and foreign body forceps. A urethral catheter was placed for 48 hours, and the patient voided well following its removal.

Key words: Urethra, Foreign Bodies, Cystoscopes, Surgical Instruments.

\section{Introduction}

Self-inserted male urethral foreign bodies are rare emergencies that urologists face occasionally. Urethral foreign body insertions are an unusual practice in which various imaginable objects are known to be inserted. Most of these episodes are associated with autoerotic stimulation, psychiatric disorders or intoxication [1]. Mannan et al. [2] reported on a series of 20 adult cases of foreign body insertions over 9 years period. Males were 1.7 times more likely to commit the act than females [2]. Autoerotic stimulation with the aid of self-inserted urethral foreign bodies has been known since time immemorial $[3,4]$. The presentation is however delayed owing to the feeling of embarrassment and guilt. Of those who seek medical attention, haematuria, dysuria, urinary frequency, strangury and urinary retention are the most common presenting features [2-5]. Dire consequences such as fulminant sepsis and death can ensue such behaviour in the event of delayed medical encounter [3]. We report on an adult male who inserted metal rods into the urethra.

\section{Case Report}

A 26 year old male was brought to hospital with blood stained undergarments. The patient was on antipsychotic drugs for a few years. He had disappeared from his house for a weeks' time and gave history of insertion of foreign objects into

\section{Corresponding Author: Dr. Ajay Kumar G}

Email: drajay707@gmail.com

Received: December 31, 2014 | Accepted: May 1, 2015 | Published Online: May 30, 2015

This is an Open Access article distributed under the terms of the Creative Commons Attribution License (creativecommons.org/licenses/by/3.0)

Conflict of interest: None declared | Source of funding: Nil | DOl: http://dx.doi.org/10.17659/01.2015.0059 
his urethra for self-sexual stimulation. He doesn't give any history of similar episodes in the past. On general examination he was well oriented to time, place and person. Local examination of his genitalia revealed blood at external urethral meatus and hard objects within the urethra could be palpated. Patient denied any history of pain in the urethra. Plain X-ray imaging revealed metallic rods within the urethra [Fig.1]. Ultrasound of KUB region failed to show the extent of metallic rods into the bladder. The decision of extraction of the foreign objects removal was planned per urethral and explained to the patient and their relatives. The foreign bodies [Fig.2] were extracted perurethra under general anaesthesia with the use of cystoscopes and foreign body forceps. Cystoscope was inseted into the patient's urethra. The tip of the metallic rods were seen in the proximal penile urethra. The tip of the metallic rods was held with forceps remover and extracted out. There was no difficulty in removal of the foreign objects. The total procedure lasted around 30 minutes. A urethral catheter was placed for 48 hours, in view of minimal damage to the urethra. The patient voided well following its removal.

\section{Discussion}

There have been several reports on self-inflicted foreign bodies in the male urethra. A wide range of foreign bodies have been inserted which include needles, pencils, ball point pens, pen lids, garden wire, copper wire, speaker wire, safety pins, keys, wire-like objects (telephone cables, rubber tubes, feeding tubes, straws, string), toothbrushes, household batteries, light bulbs, marbles, cotton tip swabs, plastic cups, thermometers, plants and vegetables (carrot, cucumber, beans, hay, bamboo sticks, grass leaves), parts of animals (leeches, squirrel tail, snakes, bones), toys, pieces of latex gloves, blue tack, intrauterine contraceptive devices (IUCD), tampons, pessaries, powders (cocaine), fluids (glue, hot wax) [2,3]. There have been several

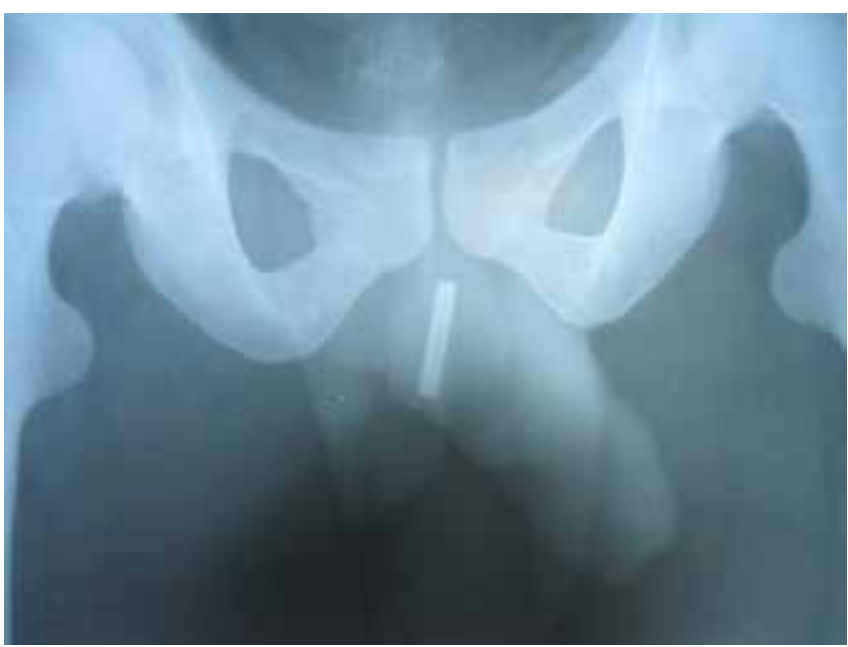

Fig.1: Plain $X$ ray showing a metallic foreign body in the region of urethra.

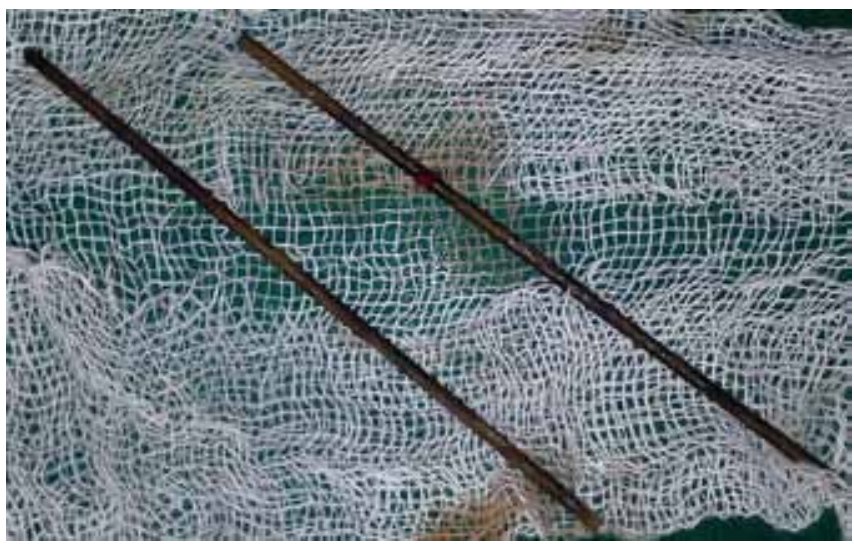

Fig.2: Extracted metallic rods from the urethra.

reasons which have been attributed to this kind of behaviour, however the main reason is of sexual nature like masturbation or sexual gratification $[1,6]$. Psychiatric illness or drug intoxication could also be other reasons.

A few psychoanalytical theories have been postulated to account for self-insertion [3]. Kenney's theory states that the initiating event is an accidentally discovered pleasurable stimulation of the urethra, which is followed by repetition of this action using objects of unknown danger, 
driven by a particular psychological predisposition to sexual gratification [7]. Some consider that urethral manipulation is a paraphilia combining sadomasochistic and fetishist elements and shows regression to a urethral stage of eroticism [3]. Several others believe that these acts are an indication of an impulsive behaviour which is selfpunishing in nature [3]. A psychiatric evaluation of such patients is strongly recommended.

The patients are usually ashamed and/ or feel guilty and therefore do not seek medical help until they do experience severe symptoms or are brought forward by close friends or relatives. The goal of treatment remains the removal of the foreign body with as little damage as possible to the urethra. Several means or procedures have been described to extract the foreign body depending on the form, size, position and mobility of the body [8]. Endoscopic removal remains the most attractive one unless open surgery becomes necessary to remove the foreign body. Naidu et al. [9] reported on extraction of fork (dining fork) via the meatus with the aide of lignocaine jelly and Rampley forceps. Ghaly et al. [10] reported on a rare case of penile foreign body lying dormant for 12 years and eventually presented with urethral discharge and urethritis like symptoms. History revealed that the patient's ex-wife had inserted cosmetic mascara through the meatus 12 years previously. The foreign body was surgically removed via open cut through the corpus spongiosum. Long term complications have been described in literature which include stricture formation, diverticulum, urinary incontinence and erectile dysfunction [6].

Regardless of the motive for placement of these foreign bodies, their extrications can be challenging and may require the creative use of urologists' surgical and occasionally the interventional radiologists' armament in their removal.

\section{Conclusion}

Foreign body retrieval is determined by its physical attributes and morphology with the aim to minimise urothelial trauma and preserve erectile function. Essentially, endo-urological extraction serves the primary means of retrieval. Cystourethoscopy is important to diagnose urothelial injuries and to ensure complete removal of foreign bodies following extraction.

\section{References}

1. Stravodimos KG, Koritsiadis G, Koutalellis G. Electrical wire as a foreign body in a male urethra : a case report. J Med Case Reports. 2009;3:49.

2. Mannan A, Anwar S, Qayyum A, Tasneem RA. Foreign bodies in the urinary bladder and their management: a Pakistani experience. Singapore Medical Journal. 2011 ;52:24-28.

3. Bedi N, El-Husseiny T, Buchholz N, Masood J. Putting lead in your pencil: self- insertion of an unusual urethral foreign body for sexual gratification. Journal of the Royal Society of Medicine Short Reports. 2010;1:18.

4. Rieder J, Brusky J, Tran V, Stern K, Aboseif S. Review of intentionally self-inflicted, accidental and iatrogenic foreign objects in the genitourinary tract. Urologia Internationalis. 2010; 84: 471-475.

5. Moon SJ, Kim DH, Chung JH, Jo JK, Son YW, Choi HY. Unusual foreign bodies in the urinary bladder and urethra due to autoerotism. International Neurourology Journal. 2010;14:186-189.

6. Mitterberger $M$, Peschel $R$, Frauscher F, Pinggera GM. Allen key completely in male urethra : a case report. Cases J. 2009;2:7408.

7. Kenney RD. Adolescent males who insert genitourinary foreign bodies; is psychiatric referral required? Urology 1988;32:1 27-129.

8. van Ophoven A, deKernion JB. Clinical 
management of foreign bodies of the genitourinary tract. J Urol. 2000;164: 274287.

9. Naidu K, Chung A, Mulcahy M. An unusual urethral foreign body. Int J Surg Case Reports.
2013.07.017.

10. Ghaly AFF, Munishankar AR, Sultana SR and Nimmo M. Case Report: foreign body in male penile urethra. Genitourin Med. 1996;72:6768. 